\title{
КРАТКОСРОЧНОЕ УПРАВЛЕНИЕ ПРИРОДНО-РЕСУРСНЫМ ПОТЕНЦИАЛОМ ПЕРМСКОГО КРАЯ С ИСПОЛЬЗОВАНИЕМ КОНЕЧНО-РАЗНОСТНЫХ МОДЕЛЕЙ
}

\section{NATURAL RESOURCE POTENTIAL SHORT-TERM MANAGEMENTUSING FINITE-DIFFERENCE MODELS OF THE PERM REGION}

\section{N. Sirotina}

Summary. In the work, finite-difference models of the Perm region natural resource potential are built. A forecast of factors was carried out, on their basis the calculation of the corresponding model values was performed. The influence of controlled and uncontrolled factors small changes of on the investigated indicator value is evaluated. A system of recommendations for the regional administration for the short-term management of the Perm region natural resource potential in order to compensate the uncontrolled factors negative influence has been developed.

Keywords: natural resource potential, finite difference model, forecasting, system of recommendations.

\section{Ввемение}

Д ля обеспечения эффективного управления устойчивым региональным развитием необходимо учитывать перспективы использования имеющихся природных ресурсов. Комплексным показателем их совокупности в рамках региона является природно-ресурсный потенциал. Кризисные явления, характерные для социально-экономических систем в современных условиях, выражаются в негативных тенденциях и цикличности. Для их коррекции, необходимо обеспечить качественное управление природно-ресурсным потенциалом региона. Это обеспечит его инвестиционную привлекательность, более динамичный экономический рост, а также позволит установить целесообразность того или иного вида хозяйственной деятельности.

Вполне закономерно, что вопросу управления природно-ресурсным потенциалом, как важнейшей компо-
Сиротина Наталья Александровна

Стариий преподаватель, ФГАОУ ВО «Пермский национальный исследовательский политехнический университет», г. Березники nsirotina117@mail.ru

Аннотация. В работе построены конечно-разностные модели природно-ресурсного потенциала Пермского края. Осуществлен прогно3 факторов, на их основе выполнен расчет соответствующих модельных значений. Исследовано влияние на уровень исследуемого показателя малых изменений управляемых и неуправляемых факторов. Выработана система рекомендаций для краевого правительства по краткосрочному управлению природно-ресурсным потенциалом Пермского края с целью компенсации негативных тенденций определяющих его факторов.

Ключевые слова: природно-ресурсный потенциал, конечно-разностная модель, прогнозирование, система рекомендаций.

нентой регионального развития, посвящено значительное число научных публикаций.

В работе [1] предпринята попытка оценить влияние природных ресурсов на международную торговлю и финансовое развития территорий, однако данный анализ осуществлен на макроуровне, а реализация указанного в статье метода статистического анализа панельных данных предполагает составную структуру объекта исследования (например, регионы Федерального округа) и не может быть использован на уровне единичного объекта. Ряд авторов разрабатывают вопрос эффективного освоения и управления ПРП посредством моделирования инвестиционных денежных потоков на примере различных стран и регионов: Африки [2], Таджикистана [3], Китая [4]. Для комплексного изучения ПРП и управления его отдельными элементами достаточно широко распространено использование геоинформационно-аналитических систем (ГИС). Так, в[5] для целей эффективного управления природными 
ресурсами рассматривается применение ГИС на территории Индии, а в [6] авторами предпринята попытка комплексной оценки природного богатства Пермского края.

Методы математического моделирования широко применимы для моделирования и прогнозирования, а, следовательно, и управления природными ресурсами территории. Так, в работе [7] представлены математические модели добычи природного газа на региональном и мировом уровнях. Однако, такой подход предполагает наличие адекватного математического критерия, подлежащего оптимизации, а также системы ограничений, а при их отсутствии данный метод практически неприменим. Исследование [8] посвящено краткому обзору существующих оптимизационных динамических моделей невозобновляемых природных ресурсов. При этом рассматриваются детерминированные модели отдельных ресурсов, что не позволяет напрямую использовать их для случая двух и более их видов, что характерно для большинства регионов. Кроме того, используемый аппарат классической теории оптимального управления требует от исследователя достаточно высокого уровня математической подготовки и может приводить к некорректным результатам применительно к стохастическим экономическим объектам. В работе [10] разработана многокритериальная многофакторная нечеткая модель частично целочисленного программирования для оптимизации распределения сельскохозяйственных водных и земельных ресурсов в условиях неопределенности. Подобное сочетание методов весьма эффективно для решения слабо формализуемых задач, однако требует разработки как специфического математического инструментария, так и программного обеспечения. В работе [10] разработана нелинейная математическая модель управления морскими природными ресурсами в форме системы нелинейных дифференциальных уравнений и выполнено ее исследование в рамках теории устойчивости динамических систем. Применение данного подхода возможно в случае, когда исследуемая система может быть адекватно описана системой обыкновенных дифференциальных уравнений.

В [11] авторы применяют детерминированную и стохастическую модели с целью оценки влияния осадков на лесные ресурсы для определения наличия и устойчивости равновесного состояния системы в зависимости от ее параметров и характеристик случайной составляющей. В [12] авторами предпринята попытка оценки регионального развития экономики в форме системы дифференциальных уравнений. В целом, детерминированные факторные модели в большей степени подходят для технических и физических систем, а поведение сложных социально - экономических си- стем не всегда адекватно описывают. Авторы в [13] рассматривают возможность применения балансовых моделей для управления потреблением водных ресурсов. Данный подход не позволяет адекватно характеризовать сложные динамические стохастические объекты, поскольку балансовые модели как правило являются статическими детерминированными.

Еще один распространенный подход к управлению природными ресурсами - моделирование с целью минимизации воздействия на окружающую среду. В [14] предпринята попытка оценки результативности мероприятий по восстановлению окружающей природной среды в рамках постановки и решения задачи линейного программирования. Авторы в [15] исследуют вопросы моделирования с целью контроля в сфере истощения запасов природных ресурсов. В работе [16] авторы предложили методику математического моделирования с целью управления отходами и ресурсами с использованием комплексного подхода.

Проведенный обзор имеющихся работ позволил установить, что вопрос управления природно-ресурсным потенциалом разработан недостаточно. Значительная часть отечественных работ являются неактуальными ввиду давности исследования. Серьезных работ, посвященные изучению ПРП Пермского края, выполненных в течение последних 20 лет в ходе проведенного обзора обнаружить не удалось. При этом оперативное планирование при использовании природных богатств органами власти является интересной задачей как с теоретической, так и прикладной точек зрения, поскольку ее решение позволяет обеспечить устойчивое развития региона. Для управления ПРП на практике необходимо решить следующие задачи:

1. Разработать математическую модель природно-ресурсного потенциала региона;

2. Осуществить сбор экономико-статистических данные на примере конкретного региона;

3. Выполнить построение разработанной математической модели на примере конкретного региона;

4. Проанализировать возможности лиц, принимающих решения (ЛПР) по противодействию негативным тенденциям неуправляемых факторов, определяющих уровень ПРП, путем варьирования управляемыми факторами на примере конкретного региона.

В качестве объекта исследования выбран ПРП Пермского края, целесообразно оценить влияние изменения управляемых и неуправляемых факторов на его величину и выработать систему рекомендаций по краткосрочному управлению природно-ресурсным потенциалом Пермского края с целью компенсации не- 
Таблица 1. Факторы, определяющие природно-ресурсный потенциал Пермского края

\begin{tabular}{|l|l|l|}
\hline$№$ & Обозначение & Наименование \\
\hline 1 & $X_{1}$ & Посевные площади сельскохозяйственных культур, тыс. га \\
\hline 2 & $X_{2}$ & Среднегодовая численность занятых в экономике, тыс. чел. \\
\hline 3 & $X_{3}$ & Инвестиции в основной капитал предприятий, млн. руб. \\
\hline 4 & $X_{4}$ & Внесение удобрений минеральные удобрения, тыс. т. \\
\hline 5 & $X_{5}$ & Внесение удобрений органические удобрения, тыс. т. \\
\hline 6 & $X_{6}$ & Стоимость основных фондов на конец года, млн. руб. \\
\hline 7 & $X_{7}$ & Лесовосстановление, тыс. га \\
\hline 8 & $X_{8}$ & Число предприятий и организаций на конец года \\
\hline 9 & $X_{9}$ & Цена нефти Вrent (среднегодовое значение), USD за баррель \\
\hline 10 & $X_{10}$ & Экспорт продукции ТЭК, млн. USD \\
\hline
\end{tabular}

гативных тенденций определяющих его факторов. Для этого необходимо:

- выполнить краткосрочный прогноз факторов, определяющих ПРП Пермского края (на 1 год);

- вычислить соответствующее прогнозное значение ПРП Пермского края (центральную точку прогноза);

- проанализировать факторы, определяющие ПРП Пермского края, и оценить их с точки зрения управляемости;

- выбрать по два наиболее значимых для региона управляемых и неуправляемых фактора;

- вычислить абсолютные значения выбранных факторов при их увеличении и уменьшении на 5\%;

- рассчитать значения ПРП при различных комбинациях значений управляемых и неуправляемых факторов, оценить, компенсирует ли положительные изменения управляемых факторов негативные изменения неуправляемых;

- в случае, если рассмотренные изменения управляемых факторов не позволяют компенсировать изменения неуправляемых, соответствующие значения следует подобрать «вручную»;

- на основании полученных расчетов разработать рекомендации по управлению ПРП Пермского края для лиц, принимающих решения.

\section{Основная часть}

Изучение имеющейся статистической информации в открытых источниках позволило установить набор показателей, определяющих уровень ПРП Пермского края (таблица 1). В связи с изменением методики расчета показателей системы национальных счетов и введения стандарта СНC 2008, а также исходя из критерия полноты необходимых статистических данных в качестве периода моделирования выбран временной интервал с 2008 по 2018 гг.
Ранее было установлено, что для прогнозирования социально-экономических процессов модели с авторегрессионными слагаемыми различных порядков (в частности, конечно-разностные модели 1 и второго порядков, КРМ-1 и КРМ-2) позволяют получить более качественные прогнозы по сравнению с традиционно используемыми линейными множественными моделями (ЛММ) [17]. При этом проведенные исследования показали, что в разные временные промежутки уровень ПРП определяют разные по составу факторы. При этом математические процедуры отбора факторов (анализ матрицы парных коэффициентов корреляции и процедура последовательного отброса факторов из уравнения) не дают удовлетворительного качества моделирования и прогнозирования. Для формирования окончательного набора факторов, определяющих ПРП в период с 2008 по 2018 г. был проведен анализ их экономической сущности с последующим исключением тесно связанных между собой или входящих один в другой в качестве составляющей части. После этого был произведен расчет коэффициентов моделей и прогнозирование на их основе; если качество полученного прогноза уступало ЛММ-прогнозу, то факторы анализировались повторно, выбиралась другая их комбинация и процедура моделирования и прогнозирования повторялась. Процедура повторялась до получения совокупности факторов, обеспечивающих удовлетворительное качество прогноза. Полученные в результате описанной процедуры уравнения КРМ-1 и КРМ-2 имеют вид соответственно

$$
\begin{aligned}
& Y_{K P M-I}\left(t_{k}\right)=-2,679+0,686 \cdot Y_{K P M-1}\left(t_{k-1}\right)+4,594 \cdot X_{I}\left(t_{k}\right)- \\
& 2,894 \cdot X_{2}\left(t_{k}\right)+2,095 \cdot X_{6}\left(t_{k}\right)- \\
& 1,876 \cdot X_{7}\left(t_{k}\right)+2,532 \cdot X_{8}\left(t_{k}\right)+3,009 \cdot X_{9}\left(t_{k}\right)+0,291 \cdot X_{10}\left(t_{k}\right)
\end{aligned}
$$

n

$Y_{K P M-2}\left(t_{k}\right)=-3,086+0,871 \cdot Y_{K P M-2}\left(t_{k-1}\right)+0,129$. $Y_{K P M-2}\left(t_{k-2}\right)++5,561 \cdot X_{1}\left(t_{k}\right)-3,678 \cdot X_{2}\left(t_{k}\right)+2,098 \cdot X_{6}\left(t_{k}\right)-$ $2,318 \cdot X_{7}\left(t_{k}\right)+2,998 \cdot X_{8}\left(t_{k}\right)++3,696 \cdot X_{9}\left(t_{k}\right)+0,270 \cdot X_{10}\left(t_{k}\right)$. 
Таблица 2. Расчет центральных точек прогноза для моделей КРМ-1 и КРМ-2

\begin{tabular}{|c|c|c|c|}
\hline № & Фактор & Метод прогноза & Прогнозна 2019 г. $\left(t_{k}=12\right)$ \\
\hline 1 & $\mathrm{X}_{1}$ & $\mathrm{TP} \mathrm{cp}=1,010$ & 0,188 \\
\hline 2 & $x_{2}$ & $X_{2}\left(t_{k}\right)=-0,1026 \cdot t_{k}+1,1724, t_{k}=12$ & $-0,161$ \\
\hline 3 & $x_{6}$ & $\mathrm{OC}_{K I I}(2018)+\mathrm{OC}_{\Pi O C T}(2019)-\overline{\mathrm{OC}_{\text {выБ }}}$ & 1,033 \\
\hline 4 & $x_{7}$ & $X_{7}\left(t_{k}\right)=0,0823 \cdot t_{k}-0,0673, t_{k}=12$ & 0,920 \\
\hline 5 & $x_{8}$ & $\mathrm{TP} c \mathrm{p}=0,982$ & 0,240 \\
\hline 6 & $X_{9}$ & $\mathrm{TP} c \mathrm{c}=0,963$ & 0,382 \\
\hline 7 & $\mathrm{X}_{10}$ & $\mathrm{TPcp}=0,931$ & 0,410 \\
\hline 8 & $Y_{K P M-1}\left(t_{k}=12\right)$ & в соответствии с уравнением (1) & 1,332 \\
\hline 9 & $Y_{K P M-2}\left(t_{k}=12\right)$ & в соответствии с уравнением (2) & 2,376 \\
\hline
\end{tabular}

Для построения краткосрочного прогноза ПРП на 2019 год в соответствии с полученными уравнениями (1) и (2) необходимы соответствующие прогнозные значения факторов. Исследование показало, что большая часть из них не имеет ярко выраженного тренда, а значит, не может быть спрогнозировано на его основании. Для таких факторов был рассчитан средний темп роста за период с 2013 по 2018 гг. и выполнен прогноз в соответствии с полученными значениями (таблица 2). Расчет прогнозного значения для фактора $X_{6}$ (стоимость основных фондов на конец года) выполним исходя из следующих соображений. Очевидно, что $\mathrm{OC}_{K \Pi}\left(t_{k}\right)=\mathrm{OC}_{H \Pi}\left(t_{k}\right)+\mathrm{OC}_{\text {Пост }}\left(t_{k}\right)-\mathrm{OC}_{\text {выБ }}\left(t_{k}\right)$, откуда $\mathrm{OC}_{\text {выБ }}\left(t_{k}\right)=\mathrm{OC}_{H \Pi}\left(t_{k}\right)+\mathrm{OC}_{\text {Пост }}\left(t_{k}\right)-\mathrm{OC}_{K \Pi}\left(t_{k}\right)$. Тогда среднюю стоимость ежегодно выбывающих объектов основных средств за период с 2008 по 2018 гг. можно рассчитать как

$$
\overline{\mathrm{OC}_{\text {ВыБ }}}=\frac{1}{11} \times\left(\mathrm{OC}_{H \Pi}(2008)+\right.
$$

$\mathrm{OC}_{\Pi О С T}(2008)-\mathrm{OC}_{K \Pi}(2008)+\mathrm{OC}_{H \Pi}(2009)+$

$\mathrm{OC}_{\Pi О С T}(2009)-\mathrm{OC}_{K \Pi}(2009)+\mathrm{OC}_{H \Pi}(2010)++\mathrm{OC}_{\Pi О С T}(2010)-$ $\left.\mathrm{OC}_{K \Pi}(2010)+\ldots+\mathrm{OC}_{H \Pi}(2018)+\mathrm{OC}_{\text {ПОСТ }}(2018)-\mathrm{OC}_{K \Pi}(2018)\right)$.

С учетом того, что суммарная стоимость основных средств на начало периода $\mathrm{OC}_{H \Pi}\left(t_{k}\right)$ равна суммарной стоимости основных средств на конец предшествующего периода $\mathrm{OC}_{K I}\left(t_{k-1}\right)$, т.е. $\mathrm{OC}_{H \Pi}(2009)=\mathrm{OC}_{K \Pi}(2008)$, полученное выражение можно переписать как

$$
\begin{aligned}
& \overline{\mathrm{OC}_{\text {ВыБ }}}=\frac{1}{11} \times\left(\mathrm{OC}_{\text {КП }}(2007)+\mathrm{OC}_{\text {ПОСТ }}(2008)-\right. \\
& \mathrm{OC}_{K \Pi}(2008)+\mathrm{OC}_{K \Pi}(2008)+\mathrm{OC}_{\Pi О C T}(2009)- \\
& \mathrm{OC}_{K \Pi}(2009)+\mathrm{OC}_{K \Pi}(2009)+\mathrm{OC}_{\Pi О C T}(2010)- \\
& \mathrm{OC}_{K \Pi}(2010)++\ldots+\mathrm{OC}_{K \Pi}(2017)+\mathrm{OC}_{\Pi О С T}(2018)- \\
& \left.\mathrm{OC}_{K \Pi}(2018)\right)=\left(\mathrm{OC}_{K \Pi}(2007)+\mathrm{OC}_{\Pi О C T}(2008)+\right. \\
& \mathrm{OC}_{\text {ПОСТ }}(2009)++\mathrm{OC}_{\text {ПОСТ }}(2010)+\ldots+\mathrm{OC}_{\text {ПОСТ }}(2018)- \\
& \left.\mathrm{OC}_{K I}(2018)\right) / 11 \text {, }
\end{aligned}
$$

откуда окончательно получим

$$
\overline{\mathrm{OC}_{\text {ВыБ }}}=\frac{1}{11} \cdot\left(\mathrm{OC}_{\text {КП }}(2007)-\mathrm{OC}_{\text {КП }}(2018)+\sum_{\mathrm{t}_{\mathrm{k}}=2008}^{2018} \mathrm{OC}_{\text {ПОСТ }}\left(t_{k}\right)\right) \approx
$$

198847,1 млн. руб.

Анализ динамики ежегодных инвестиций в объекты основных средств $X_{3}\left(t_{k}\right)=\mathrm{OC}_{\text {Пост }}\left(t_{k}\right)$ в период с 2008 по 2018 гг. на территории Пермского края позволил установить их линейную тенденцию вида $X_{3}\left(t_{k}\right)=12530 \cdot t_{k}+116577$ с надежностью $85,96 \%$. Тогда $X_{3}(2019)=\mathrm{OC}_{\text {ПОСТ }}(2019)=12530 \cdot 12+116577=266937$ млн. руб.

Тогда расчетное значение стоимости объектов основных средств на конец 2019 г. составит

$O C_{K \Pi}(2019) \approx O C_{H \Pi}(2019)+O C_{\Pi о с T}(2019)-\overline{\mathrm{OC}_{\text {выБ }}}=$ $\mathrm{OC}_{К \Pi}(2018)+\mathrm{OC}_{\text {ПОСТ }}(2019)-\overline{\mathrm{OC}}_{\text {выб }}==3576306+266937-$ $198847,1=3644395,9$ млн. руб.

Выполним нормирование полученного значения:

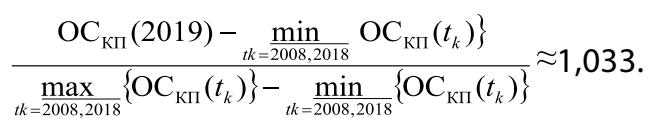

На основании полученных значений факторов были рассчитаны центральные точки прогноза - значения ПРП Пермского края в соответствии с уравнениями (1) и (2) при $t_{k}=12, Y_{K P M-1}\left(t_{k}=12\right)$ и $Y_{K P M-2}\left(t_{k}=12\right)$ (таблица 2).

Возможность управления факторами, определяющими ПРП, может быть установлена на основании их качественного анализа. Так, к управляемым факторам можно отнести:

- $X_{1-}$ посевные площади сельскохозяйственных культур, тыс. га;

- $X_{3}$ - инвестиции в основной капитал предприятий, млн. руб.;

- $X_{4}$ - внесение удобрений минеральные удобрения, тыс. т.; 
Таблица 3. Изменение уровней определяющих ПРП факторов на 5\%

\begin{tabular}{|l|l|l|l|}
\hline Фактор & $X-5 \%$ & $\mathrm{X}$ & $X+5 \%$ \\
\hline $\mathrm{X}_{1}$ & 0,1767 & 0,1860 & 0,1953 \\
\hline $\mathrm{X}_{2}$ & $-0,0559$ & $-0,0588$ & $-0,0617$ \\
\hline$X_{6}\left(\right.$ в размере $\left.X_{3}\right)$ & 1,0264 & 1,0328 & 1,0399 \\
\hline$X_{9}$ & 0,3632 & 0,3824 & 0,4015 \\
\hline
\end{tabular}

Таблица 4. Абсолютные и относительные КРМ прогнозы ПРП при малых изменениях неуправляемых факторов

\begin{tabular}{|l|l|l|l|l|l|l|}
\hline & KPM-1 & $X_{2}$ & $X_{2}+5 \%$ & $X_{2}-5 \%$ & $X_{2}$ & $X_{2}+5 \%$ \\
\hline$X_{9}-5 \%$ & $\begin{array}{l}X_{2}-5 \% \\
(-266\end{array}$ & $\begin{array}{l}1,275 \\
(-4,317 \%)\end{array}$ & $\begin{array}{l}1,283 \\
(-3,679 \%)\end{array}$ & $\begin{array}{l}2,294 \\
(-3,429 \%)\end{array}$ & $\begin{array}{l}2,305 \\
(-2,974 \%)\end{array}$ & $\begin{array}{l}2,316 \\
(-2,519 \%)\end{array}$ \\
\hline$X_{9}$ & 1,324 & 1,332 & 1,341 & 2,365 & 2,376 & 2,387 \\
$(-0,639 \%)$ & $(0,0 \%)$ & $(0,639 \%)$ & $(-0,455 \%)$ & $(0,0 \%)$ & $(0,455 \%)$ \\
\hline$X_{9}+5 \%$ & 1,381 & 1,390 & 1,398 & 2,436 & 2,446 & 2,457 \\
$(2,519 \%)$ & $(2,974 \%)$ & $(3,429 \%)$ \\
\hline
\end{tabular}

- $X_{5}$ - внесение удобрений органические удобрения, тыс. т;

- $X_{7}$ - лесовосстановление, тыс. га.

K неуправляемым факторам относятся:

- $X_{2}$ - среднегодовая численность занятых в экономике, тыс. чел.;

- $X_{6}$ - стоимость основных фондов на конец года, млн. руб.;

- $X_{8}$ - число предприятий и организаций на конец года;

- $X_{9}$ - цена нефти Brent (среднегодовое значение), USD за баррель;

- $X_{10}$ - экспорт продукции TЭК, млн. USD.

При этом, как отмечалось ранее, $X_{6}\left(t_{k}\right)=X_{6}\left(t_{k-1}\right)+X_{3}\left(t_{k}\right)-$ OC выбыло за период, тогда $X_{6}$ можно считать управляемым в сумме $X_{3}$. Тогда управляемыми факторами в (1) и (2) будут $X_{1}, X_{6}$ в сумме $X_{3}, X_{7}$; неуправляемые факторы в (1) и (2) $-X_{2}, X_{8}, X_{9}, X_{10}$. Возможность оперативного управления размерами посевных площадей $\left(X_{l}\right)$ определяется активно реализуемыми программами поддержки сельхозпроизводителей на территории Пермского края в рамках реализации стратегии импортозамещения. Наиболее естественным с точки зрения управления является фактор инвестиций в основной капитал $\left(X_{3}\right)$; поскольку он не входит в модели (1) и (2) явно, а лишь в составе $X_{6}$, то целесообразно выбрать в качестве управляющего фактора $X_{3}$ в составе $X_{6}$.Фактор лесовосстановления $\left(X_{7}\right)$ является одним из важнейших для целей обеспечения устойчивого развития региона [18], данный вид деятельности в Пермском крае активно реализуется на практике, однако эффект от него не может быть получен в краткосрочной перспективе. Таким образом, в качестве управляемых факторов выбраны $X_{1}$ и $X_{3}$ в составе $X_{6}$.

Наиболее значимыми неуправляемыми факторами являются: $X_{2}$ - среднегодовая численность занятых в экономике и $X_{9}$ - цена нефти Brent. Показатель численности занятых в большей степени определяется экономической конъюнктурой и социальной ответственностью бизнеса, и в меньшей степени - политикой региональных властей, соответственно, управление им затруднительно. Стоимость нефти определяется взаимодействием мирового спроса и предложения, на ее уровень до определенной степени могут повлиять федеральные, но не региональные власти.

Для оценки влияния изменения факторов на уровень ПРП рассчитаем увеличение и уменьшение прогнозных значений выбранных управляемых и неуправляемых факторов на 5\% (таблица 3).

Их наличие позволяет определить соответствующие уровни ПРП по каждому из уравнений (1) и (2) (таблицы 4 и 5).

Поскольку положительной динамикой ПРП является рост, то наихудший сценарий из рассмотренных соответствует снижению обоих неуправляемых факторов на $5 \%$, т.е. 
Таблица 5. КРМ прогнозы ПРП при малых изменениях управляемых факторов

\begin{tabular}{|c|c|c|c|c|c|c|}
\hline & \multicolumn{3}{|c|}{ КРМ-1 1 год } & \multicolumn{3}{|c|}{ КРМ-2 1 год } \\
\hline & $\begin{array}{l}X_{6} \\
\left(X_{3}-5 \%\right)\end{array}$ & $\begin{array}{l}X_{6} \\
\left(X_{3}=\text { const }\right)\end{array}$ & $\begin{array}{l}X_{6} \\
\left(X_{3}+5 \%\right)\end{array}$ & $\begin{array}{l}X_{6} \\
\left(X_{3}-5 \%\right)\end{array}$ & $\begin{array}{l}X_{6} \\
\left(X_{3}=\text { const }\right)\end{array}$ & $\begin{array}{l}X_{6} \\
\left(X_{3}+5 \%\right)\end{array}$ \\
\hline$X_{1}-5 \%$ & $\begin{array}{l}1,276 \\
(-4,217 \%)\end{array}$ & $\begin{array}{l}1,290 \\
(-3,206 \%)\end{array}$ & $\begin{array}{l}1,305 \\
(-2,194 \%)\end{array}$ & $\begin{array}{l}2,311 \\
(-2,745 \%)\end{array}$ & $\begin{array}{l}2,324 \\
(-2,176 \%)\end{array}$ & $\begin{array}{l}2,338 \\
(-1,608 \%)\end{array}$ \\
\hline$x_{1}$ & $\begin{array}{l}1,319 \\
(-1,012 \%)\end{array}$ & $\begin{array}{l}1,332 \\
(0,0 \%)\end{array}$ & $\begin{array}{l}1,347 \\
(1,012 \%)\end{array}$ & $\begin{array}{l}2,362 \\
(-0,568 \%)\end{array}$ & $\begin{array}{l}2,376 \\
(0,0 \%)\end{array}$ & $\begin{array}{l}2,389 \\
(0,568 \%)\end{array}$ \\
\hline$X_{1}+5 \%$ & $\begin{array}{l}1,362 \\
(2,194 \%)\end{array}$ & $\begin{array}{l}1,375 \\
(3,206 \%)\end{array}$ & $\begin{array}{l}1,390 \\
(4,217 \%)\end{array}$ & $\begin{array}{l}2,414 \\
(1,608 \%)\end{array}$ & $\begin{array}{l}2,427 \\
(2,176 \%)\end{array}$ & $\begin{array}{l}2,441 \\
(2,745 \%)\end{array}$ \\
\hline
\end{tabular}

Таблица 6. Зависимость значения $\Delta Y_{\mathrm{KPM}-1}$ и $\Delta Y_{\mathrm{KPM-2}}$ от темпа роста Х3

\begin{tabular}{|l|l|l|l|l|l|l|l|l|l|}
\hline Темп роста $X_{3}$ & 1,050 & 1,060 & 1,070 & 1,080 & 1,086 & 1,090 & 1,100 & 1,110 & 1,120 \\
\hline$\Delta Y_{\text {КРМ }-1}$ & 0,010 & 0,007 & 0,004 & 0,002 & 0,000 & $-0,001$ & $-0,004$ & $-0,006$ & $-0,009$ \\
\hline Темп роста $X_{3}$ & 1,050 & 1,060 & 1,070 & 1,080 & 1,090 & 1,100 & 1,110 & 1,120 & 1,130 \\
\hline$\Delta Y_{\text {КРМ-2 }}$ & 0,016 & 0,014 & 0,011 & 0,008 & 0,050 & 0,003 & 0,000 & $-0,003$ & $-0,005$ \\
\hline
\end{tabular}

$Y_{K P M-1}\left(t_{k}=12, X_{2}-5 \%, X_{9}-5 \%\right)=1,266, Y_{K P M-2}\left(t_{k}=12, X_{2}\right.$ $\left.5 \%, X_{9}-5 \%\right)=2,294$. Тогда снижение уровня ПРП относительно его центральной точки прогноза составит

$$
\begin{aligned}
& \Delta Y_{\text {КРМ }-1}^{\text {неупр }}=1,266-1,322=-0,066, \\
& \Delta Y_{\text {КРМ }-2}^{\text {неупр }}=2,294-2,376=-0,081 .
\end{aligned}
$$

Поскольку положительной динамикой ПРП является рост, то наилучший сценарий из рассмотренных соответствует росту обоих управляемых факторов на 5\%, т.e. $Y_{K P M-I}\left(t_{k}=12, X_{I}+5 \%, X_{6}\left(X_{3}+5 \%\right)\right)=1,390, Y_{K P M-2}\left(t_{k}=12\right.$, $\left.X_{1}+5 \%, X_{6}\left(X_{3}+5 \%\right)\right)=2,441$. Тогда увеличение уровня ПРП относительно его центральной точки прогноза составит

$$
\begin{aligned}
& \Delta Y_{\mathrm{KРM-1}}^{\text {упр }}=1,390-1,322=0,058, \\
& \Delta Y_{\mathrm{KРM-2}}^{\text {Упр }}=2,441-2,376=0,065 .
\end{aligned}
$$

Очевидно, что отрицательная динамика ПРП в результате снижения неконтролируемых факторов не компенсируется его положительной динамикой в результате роста контролируемых факторов. Понятно, что в результате изменения $X_{3}$ на $5 \% X_{6}$ меняется менее чем на $5 \%$. В связи с этим необходимо рассчитать, как должен измениться $X_{3}$, чтобы обеспечить нужную положительную динамику ПРП, т.е. чтобы величина

$$
\Delta Y_{\mathrm{KPM-1}}=\Delta Y_{\mathrm{KPM}-1}^{\mathrm{yпр}}+\Delta Y_{\mathrm{KPM}-1}^{\text {неупр }}=0 \text { и }
$$

$\Delta Y_{\mathrm{KPM-2}}=\Delta Y_{\mathrm{KPM}-2}^{\mathrm{yпр}}+\Delta Y_{\mathrm{KPM}-2}^{\mathrm{Hеупр}}=0$ (таблица 6).

Таким образом, для компенсации величины $\Delta Y_{\mathrm{KPM}-1}^{\text {неупр }}$ соответствующим значением $\Delta Y_{\mathrm{KPM}-1}^{\text {упр }}$ необходимо увеличение суммы годовых инвестиций в основной капитал на 8,6\%; для компенсации величи-

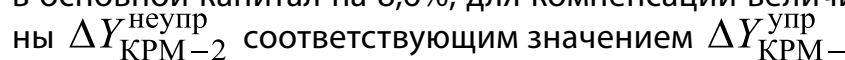
необходимо увеличение суммы годовых инвестиций в основной капитал на 11,0\% при увеличении площади посевных площадей на 5\%.

По результатам проведенного исследования можно сделать следующие выводы.

1. Отрицательной динамикой неуправляемых факторов $X_{2}$ (среднегодовая численность занятых в экономике) и $X_{9}$ (цена нефти Brent) является снижение. При уменьшении факторов на 5\% происходит уменьшение ПРП на 4,956\% для КРМ-1 и на 3,429\% для КРМ-2.

2. Положительной динамикой управляемых факторов $X_{1}$ (размеры посевных площадей) и $X_{3}$ в составе $X_{6}$ (инвестиций в основной капитал в составе стоимости основных фондов на конец года) является рост. При увеличении факторов на 5\% происходит рост ПРП на 4,217\% для КРМ-1 и на $2,745 \%$ для КРМ-2.

3. Прирост ПРП в результате положительного изменения управляемых факторов $X_{1}$ и $X_{3}$ в составе $X_{6}$ на $5 \%$ не компенсирует его снижение в результа- 
те негативного изменения неуправляемых факторов $X_{2}$ и $X_{9}$ на $5 \%$.

4. Поскольку обеспечить увеличение посевных площадей быстрыми темпами в краткосрочном периоде затруднительно и требует существенных затрат различных ресурсов, целесообразно добиваться нужных темпов положительной динамики ПРП посредством управления суммой годовых инвестиций в объекты основных средств, т.е. $X_{3}$ в составе $X_{6}$.

5. Для обеспечения $\Delta Y_{\mathrm{KPM-1}}=0$ достаточно обеспечить прирост годовых инвестиций в основной капитал в размере 8,6\%; для обеспечения $\Delta Y_{\text {КРМ-2 }}=0$ достаточно обеспечить прирост годовых инвестиций в основной капитал в размеpe $11,0 \%$. Соответствующие суммы в абсолютном выражении составляют 24,024 и 29,363 млрд. руб.

Таким образом, ЛПР на уровне краевых органов власти для противодействия негативным тенденци- ям неуправляемых факторов, определяющих уровень ПРП, можно порекомендовать стимулировать инвестиционную активность региональных хозяйствующих субъектов. Поскольку по официальным данным [19] совокупные сборы налога на прибыль в 2019 г. составили порядка 60 млрд. руб., то оставшаяся в распоряжении предприятий сумма чистой прибыли составляет порядка 300 млрд. руб., т.е. ее достаточно для инвестиционной деятельности в требуемых объемах. Кроме того, краевые власти могут возместить соответствующие затраты предприятиям, активно реализующим инвестиционные проекты, в виде льгот по налогу на прибыль. Несмотря на некоторое сокращение доходной части регионального бюджета в краткосрочной перспективе, инвестиционная активность будет способствовать не только росту ПРП, но и экономики в целом, в частности, увеличению занятости населения, росту ВРП, строительства, машиностроения. Это позволяет заключить, что ЛПР могут обеспечить устойчивое развитие ПРП региона даже в неблагоприятных внешних и внутренних условиях.

\section{ЛИТЕРАТУРА}

1. Redmond T., Nasir M.A. Role of natural resource abundance, international trade and financial development in the economic development of selected countries // Resources Policy - 2020 - Volume 66. - P. 65-78.

2. Buur L., Pedersen R., Nystrand M., Macuane J., Jacob T. The politics of natural resource investments and rights in Africa: A theoretical approach // The Extractive Industries and Society. — 2020.- Volume 7.- P. 918-930.

3. Ходиев Д.А., Хофизов Ф.А. Моделирование и прогнозирование привлечения инвестиций для освоения природно-ресурсного потенциала региона // Вестник Таджикского национального университета. - 2018. - № 6. - С. 30-36.

4. Hussain J., Zhou K, Guo S., Khan A. Investment risk and natural resource potential in "Belt \& Road Initiative" countries: A multi-criteria decision-making approach // Science of The Total Environment. — 2020._ Volume 723.137981.

5. Kumar N., YamaçS.S., Velmurugan A., Applications of Remote Sensing and GIS in Natural Resource Management // Journal of the Andaman Science Association. - 2015. - Volume 20(1).-P.1-6.

6. Красильников П.А. Геоинформационное обеспечение экономической оценки природно-ресурсного потенциала территорий Пермского края // Экономика региона. - 2009.— № 1.-С. 143-151.

7. Горячев А.А. Моделирование добычи в мировых и региональных оптимизационных газовых моделях // Проблемы экономики и управления нефтегазовым комплексом. - 2015. - № 6.- С. 51-58.

8. Кузнецов Ю.А., Семенов А.В., Власова М.Н. Математическое моделирование оптимального использования невозобновимых природных ресурсов // Экономический анализ: теория и практика. — 2012.— № 32.—C. 45-57.

9. Li M., Fu Q., Singh P.V., Liu D., Li T. Managing agricultural water and land resources with tradeoff between economic, environmental, and social considerations: A multi-objective non-linear optimization model under uncertainty // Agricultural systems. — 2020.— Volume 178. — 102685.

10. Biswas M.H., Hossain M.R., Mondal M.K. Mathematical Modeling Applied to Sustainable Management of Marine Resources // Procedia Engineering.2017. - Volume 194.-P.337-344.

11. Lata K., Misra A.K. The influence of forestry resources on rainfall: A deterministic and stochastic model // Applied Mathematical Modelling, 2020.— Volume 81.- P. 673-689.

12. Курышев Н.И. Модель и способ оценки эколого-экономического состояния регионов // Вестник кибернетики. - 2005. - № 4. - С. 149-158.

13. Кудрявцева 0.В. Математические модели анализа потребления природных ресурсов // Вестник Московского университета. Серия 6: Экономика. 2007. — № 3.- - C. 67-87.

14. Бурцев С.В. Экономико-математическая модель оценки эффективности мероприятий по восстановлению окружающей природной среды закрываемых угольных предприятий // Горный информационно-аналитический бюллетень (научно-технический журнал). - 2012.— № 1.—C. $357-359$.

15. Потравный И.М., Новоселова А.Л., Алныкина Е.М. Модели истощения природных ресурсов и оценки прошлого ущерба от загрязнения окружающей среды // Плехановский научный бюллетень. - 2015.— № 2.- C. 53-78.

16. Соловьева Н.В., Худошина М.Ю. Комплексный подход к моделированию управления ресурсами и отходами в системе «природа-техносфера» с целью минимизации воздействия на окружающую среду // Безопасность жизнедеятельности. — 2012.— № 2.— C. 33-40. 
17. Сиротина Н.А., Копотева А.В., Затонский А.В. Оценка вклада горнодобывающей отрасли в природно-ресурсный потенциал региона // Горный информационно-аналитический бюллетень (научно-технический журнал). - 2020.— № 8. - С. 163-178.

18. Лесной план Пермского края на 2018-2027 годы. https://priroda.permkrai.ru/timberraw/les_plan/ (дата обращения 25.04.2021)

19. https://www.permkrai.ru/news/minfin-prikamya-byudzhet-2019-goda-profitsitnyy/

(c) Сиротина Наталья Александровна (nsirotina117@mail.ru).

Журнал «Современная наука: актуальные проблемы теории и практики»

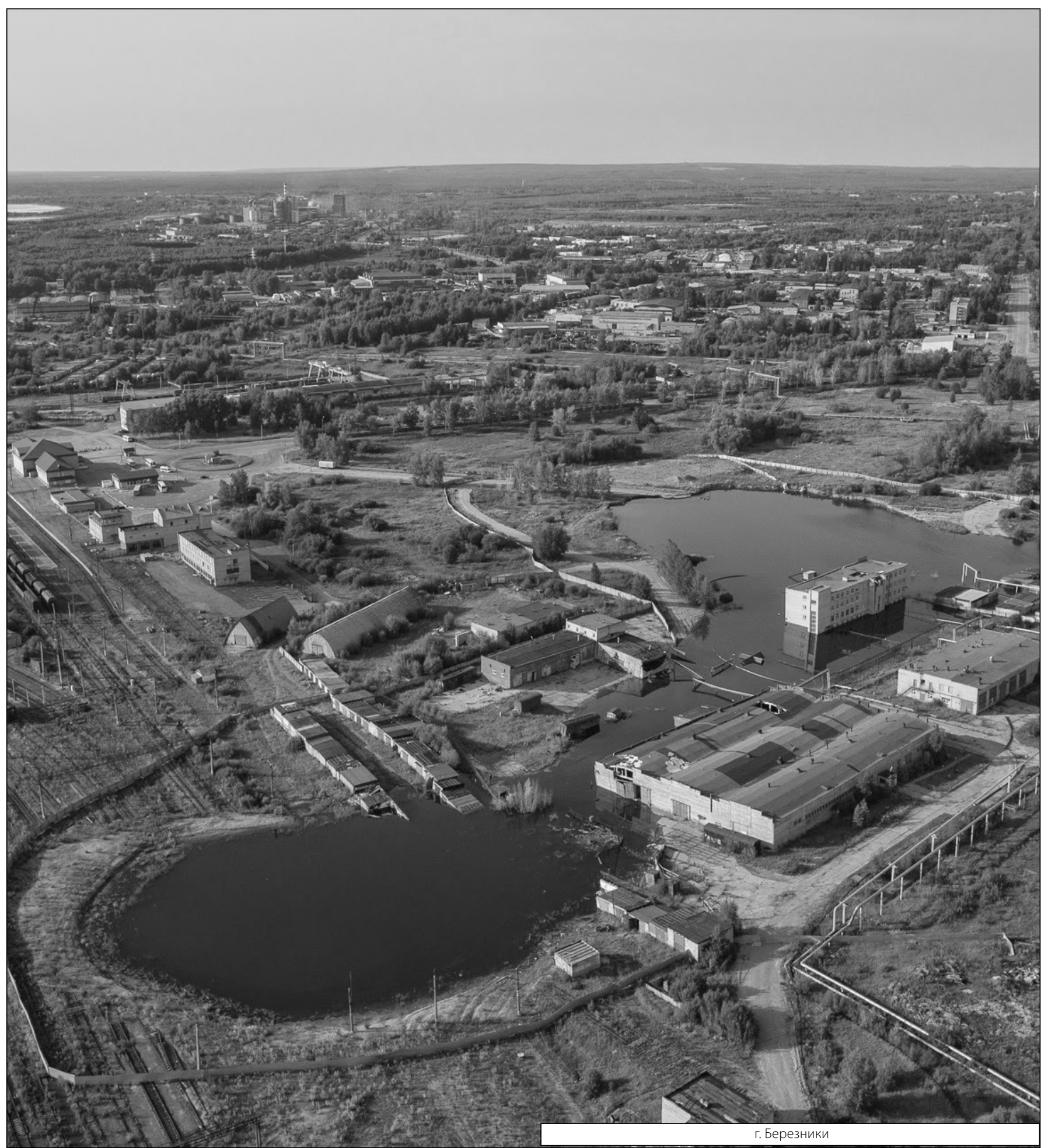

